\title{
Evaluación y Estandarización de la Calidad del Espermiograma: Nuevos Límites Inferiores de Referencia
}

\author{
Evaluation and Standardization of the Quality of Semen: New Lower Reference Limit
}

"Espinoza-Navarro, O. \& **'Sarabia, L.

ESPINOZA-NAVARRO, O. \& SARABIA, L. Evaluación y estandarización de la calidad del espermiograma: nuevos límites inferiores de referencia. Int. J. Morphol., 29(3):885-890, 2011.

RESUMEN: El análisis seminal o espermiograma es uno de los parámetros más usados en la evaluación de la fertilidad masculina. La OMS (WHO, 2010), presentó el $5^{\circ}$ Manual para el examen y procesamiento del semen humano, documento que fue analizado durante el primer taller de estandarización del análisis seminal (PLEAS), realizado en Santiago de Chile, mayo del 2010. Posteriormente se aplicaron los nuevos valores indicados como "límite de referencia inferior" (LIR), en el estudio del análisis seminal realizados por varios autores (2003 al 2010). Los resultados obtenidos indican que el $81 \%$ de los investigadores latino americanos creen que el nuevo manual estandariza mejor la concentración espermática, un $96 \%$ está de acuerdo con la nueva subclasificación en la motilidad espermática en progresiva (A), no progresiva (B) e inmóviles (C). El 68\% estima que el mejor instrumental de recuento es la cámara de Neubauer. Respecto a los controles de calidad solo el 18\% realiza controles de calidad externa. El 100\% de los investigadores estima conveniente realizar continuos talleres de estandarización. Respecto a la aplicación de los LIR en las poblaciones en estudio, todos ellos cumplirían con los estándares actuales para ser considerada una población con capacidad de fertilidad. Sin embargo varios autores opinan que una nueva versión del manual OMS, debe realizarse urgentemente para estandarizar mejor la concentración espermática (15 millones por $\mathrm{mL}$ ) y la morfología según criterios estrictos (4\%), valores de referencia que consideran muy bajos.

PALABRAS CLAVE: Análisis seminal; Espermiograma; Fertilidad masculina; Límite de referencia inferior.

\section{INTRODUCCIÓN}

En la década de los 50, MacLeod \& Gold (1953) comenzaron a comparar los seminogramas de personas fértiles con pacientes con problemas para concebir un hijo, encontrando una estrecha relación entre la concentración espermática, la motilidad de ellos y su influencia en la capacidad fecundante de los varones en estudio. El semen normal es una mezcla de espermatozoides suspendidos en las secreciones testiculares y epididimarias, que luego se combinan con las secreciones prostáticas, de las vesículas seminales y de las glándulas bulbouretrales. El análisis seminal por tanto permite dar información sobre la espermatogénesis y la condición fisiológica de los tejidos comprometidos (Aitken, 2010).

En los últimos años el análisis seminal continúa siendo una de las mejores herramientas en la investigación de la fertilidad masculina y la que brinda la mejor información previa para evaluar la calidad reproductiva de los varones.
Sin embargo la determinación de los parámetros seminales deben seguir estrictas normas de control, para no perder su objetividad clínica (Pacey, 2010; Sarabia \& Munuce, 2011). Otros investigadores clínicos además proponen la incorporación de otros test complementarios (Agarwal \& Said, 2011).

Los trabajos realizados por Cortés-Gutiérrez et al. (2007) y Cruz et al. (2010), determinan que aparte de la concentración, motilidad y morfología espermática, se debería incorporar el estudio sobre la integridad del ADN espermático, ya que la fragmentación en las cadenas simples o dobles del ADN, son muy frecuentes en pacientes no fértiles.

Los estudios además consideran de relevante importancia la edad de los pacientes o donantes debido a que los autores han encontrado una directa relación entre pacientes

\footnotetext{
* Department of Biology, Faculty of Sciences, Universidad de Tarapacá. Arica-Chile.

${ }^{* *}$ Laboratory of spermiogram, ICBM Faculty of Medicine, Universidad de Chile, Santiago-Chile.
} 
de mayor edad con el aumento de daño en el ADN espermático, lo que explicaría la reducción de la fertilidad en estos varones sobre los 40 años de edad, observándose disminución en la concentración espermática y la motilidad con aumento de espermatozoides inmóviles (Cánovas et al., 2008; Horta et al., 2011).

Por lo tanto hay que considerar que las características del semen varían según la edad, patologías asociadas, exposición a diversos contaminantes naturales y antropogénicos, sector geográfico, luminosidad, consumo de alcohol y tabaco (Martini et al., 2004; Ramlau-Hansen et al., 2007; Gao et al., 2007; Levitas et al., 2007; Cánovas et al.: Imai et al., 2010; Ellekilde Bonde, 2010).

El objetivo de este trabajo fue analizar a la luz de las nuevas y actuales referencias (WHO, 2010), los parámetros en la calidad seminal y su relación en la fecundidad y en la investigación de la salud reproductiva. Para lo cual se hace imperativo homogenizar este examen en los laboratorios andrológicos o de investigación en Latino América y el mundo.

\section{MATERIAL Y MÉTODO}

Se realizó un análisis de cómo 28 laboratorios latinoamericanos en América Latina realizan e interpretan el espermiograma, según las directrices que propone la OMS en su $5^{\circ}$ Manual para el Examen y procesamiento de semen humano (WHO, 2010). Este análisis fue el producto del trabajo realizado durante el Primer Taller del Programa Latino Americano para la estandarización del análisis seminal (PLEAS), Mayo de 2010, Santiago de Chile.

Lo anterior se complementó con una actualizada y extensa revisión bibliográfica, acerca de los diferentes com- ponentes del examen seminal. Finalmente se compararon los resultados obtenidos en diversos laboratorios del mundo (2003 al 2008), con los resultados obtenidos en una población sana de la ciudad de Arica-Chile (2010), frente a los actuales "Límites Inferiores de Referencia" (LIR).

\section{RESULTADOS}

En la Tabla I se observa la apreciación de los investigadores latino americanos sobre los parámetros propuestos por la $5^{\circ}$ edición OMS (WHO, 2010). El 96\% de los participantes está de acuerdo con la nueva subclasificación de la motilidad espermática en: progresivos (A), no progresivos (B) e inmóviles (C). Sin embargo en relación a los sistemas de controles de calidad, el 57\% realiza controles internos dentro de sus laboratorios, pero solo el $18 \%$ aplica controles de calidad externos. El uso de la cámara de Neubauer sigue siendo el instrumental más usado para el recuento espermático (68\%). El 100\% de los investigadores considera pertinente realizar continuos talleres de estandarización seminal.

En la Tabla II se muestran los valores de referencia según la OMS (WHO, 1999) y los actuales "Límites Inferiores de Referencia" (LIR), según el nuevo manual OMS (WHO, 2010).

En la Tabla III se observan los resultados de los parámetros seminales encontrados en poblaciones jóvenes según investigaciones realizadas en varios laboratorios del mundo (2003 al 2008), y su comparación con los resultados obtenidos en una población aparentemente sana de Arica-Chile (2010). Se observa que tanto la población Argentina como la de Chile al igual que todas las otras poblaciones en estudio podrían considerarse como potencialmente fértil según los actuales "Límites Inferiores de Referencia" (LIR), OMS-2010

Tabla I. Resultados de encuesta sobre los niveles de aceptación de las recomendaciones OMS-2010 a los investigadores asistentes al Primer taller de estandarización seminal en América Latina (PLEAS, 2010).

\begin{tabular}{lc}
\hline \multicolumn{1}{c}{ Indicador referencial } & $\begin{array}{c}\text { Porcentaje de aceptación } \\
\text { Manual OMS-2010 }\end{array}$ \\
\hline ¿El nuevo manual estandariza mejor la concentración espermática? & 81 \\
¿La nueva clasificación espermática en: Progresivos (A), no progresivos (B) e inmóviles (C), mejora la \\
reproducibilidad entre los operadores? & 96 \\
¿Los nuevos valores de "límites inferiores de referencia" (LIR), mejora la e valuación de varón fértil? & 44 \\
¿Realiza talleres de control interno? & 57 \\
¿Realiza talleres de control Externos? & 18 \\
¿Cuál es el instrumental que usa para medir concentración espermática? & 68 \\
Cámara Neubauer & 39 \\
Cámara de Makler & \\
¿Considera pertinente realizar continuos talleres de estandarización? & 100 \\
\hline
\end{tabular}


Tabla II. Muestra la comparación entre los valores de referencia OMS-1999 y los límites de referencia inferiores (LIR) según Manual OMS-2010.

\begin{tabular}{lcc}
\hline & $\begin{array}{c}\text { OMS-1999 } \\
\text { (4ta Edición) }\end{array}$ & $\begin{array}{c}\text { OMS-2010 } \\
\text { (5ta Edición) }\end{array}$ \\
\hline $\mathrm{pH}$ & Valor de referencia & LIR \\
volumen & $7,2-7,8$ & $\geq 7,2$ \\
Concentración es permática & $2 \mathrm{ml}$ & $1,5 \mathrm{ml}(1,4-1,7)$ \\
Concentración total & $20 \times 10^{6} / \mathrm{mL}$ & $15 \times 106 / \mathrm{ml}(12-15)$ \\
Motilidad total (progresivos + no progresivos) & $40 \times 106$ & $39 \times 106(33-46)$ \\
Motilidad progresiva & & $40 \%(38-42)$ \\
Viabilidad & $50 \%$ & $32 \%(31-34)$ \\
Formas normales & $75 \%$ & $58 \%(55-63)$ \\
Leucocitos & $15 \%$ & $4 \%(3-4)$ \\
\hline
\end{tabular}

Tabla III. Descripción de los parámetros seminales en población joven en América y el mundo, período 2003 al 2010.

\begin{tabular}{|c|c|c|c|c|c|c|}
\hline País & $\mathbf{n}$ & $\begin{array}{l}\text { Edad } \\
\text { (años) }\end{array}$ & $\begin{array}{c}\text { Concentración } \\
\text { Millones por mL }\end{array}$ & Motilidad & Morfología & Autor \\
\hline Referencia & & & $15 \times 106 / \mathrm{ml}$ & $40 \%(38-42)$ & $4 \%(3-4)$ & WHO-2010 \\
\hline Chile-Arica & 102 & $18-30$ & 62,8 & 42,2 & 15 & Espinoza-Navarro et al., 2010 \\
\hline Argentina & 3976 & 33,2 & 50,9 & 44 & 11 & Martini et al., 2004 \\
\hline USA & 408 & 33,3 & 136 & 62,3 & 8,1 & Chen et al., 2003 \\
\hline Dinamarca & 1702 & 19 & 48 & 65,5 & 10,5 & Jensen et al., 2007 \\
\hline Israel & 1751 & $25-30$ & 64,3 & 42,3 & 8,41 & Levitas et al., 2007 \\
\hline Singapur & 48 & $<40$ & 72,3 & 47 & 7,9 & Ho et al., 2007 \\
\hline Suiza & 34 & 34 & 60 & 42 & 8 & Crazzolara et al., 2007 \\
\hline China & 276 & $25-30$ & 70 & 50 & ------ & Gao et al., 2007 \\
\hline España & 309 & ------ & 79 & 56 & ------ & Aulesa et al., 2007 \\
\hline
\end{tabular}

\section{DISCUSIÓN}

Un $20 \%$ de las parejas en edad reproductiva presenta problemas de esterilidad o infertilidad, fenómeno que afecta a ambos sexos, por lo tanto en la mitad de los casos el factor masculino tiene un papel relevante.

Las causas más frecuentes relacionadas con los problemas masculinos, son los defectos en la función espermática. La calidad del semen se toma como una buena medición en fecundidad masculina, toxicología reproductiva, epidemiología y riesgo de embarazo (Cooper et al., 2010), esta calidad seminal se asocia generalmente a una buena concentración, motilidad y morfología espermática (CortésGutiérrez et al.; Góngora-Rodríguez \& Fontecilla-Ramírez, 2010).
El nuevo Manual para el examen y procesamiento de semen Humano (WHO, 2010), pretende homogenizar mundialmente estos valores en todos los laboratorios e investigadores en reproducción. Esta versión recoge la información de 4500 varones de 8 países en tres continentes, lo cual asegura un trabajo de mayor rigor científico.

Uno de los mayores desafíos de este nuevo manual es la aceptación por parte de los andrólogos e investigadores en salud reproductiva. Los cambios en los referenciales de concentración y motilidad espermática son los más aceptados (81\% y $96 \%$ respectivamente). Similares conclusiones presentan actuales trabajos donde la concentración espermática resulta el parámetro más significativo de la fun- 
ción testicular. Del mismo modo la nueva clasificación en la motilidad es mucho más cuantitativa al ser analizada solo en tres grupos: Progresivos (A), No progresivos (B) y espermatozoides inmóviles (C) (Cardona Maya, 2010).

Los controles de calidad en la toma y análisis de las muestras, resulta ser deficiente, un $57 \%$ realiza controles e calidad interna y solo un $18 \%$ aplica controles de calidad externa. El uso de la cámara de Neubauer sigue siendo el instrumental más usado en el recuento espermático (68\%). Por lo tanto los intentos de comparación internacional fallan a menudo, debido especialmente a las diferencias en los métodos de análisis y reclutamiento de las muestras y de los sujetos (Cooper et al.; Lu et al., 2010).

Los nuevos valores de "límites inferiores de referencia", se analizan y se encuentra que solo al $44 \%$ de los investigadores le parecen aceptables. Esto se observa muy bien en la tabla 3 en la cual todas las poblaciones en estudio cumplirían con los estándares para ser consideradas como población aparentemente fértil. Sin embargo Menkveld (2010), estima que el valor de corte para la morfología espermática es muy bajo $(4 \%)$, lo cual se debería a la tendencia mundial en la clasificación de la morfología normal del espermatozoide, según criterios estrictos de evaluación (criterios de Tygerberg). Igual análisis hace Eliasson (2010) respecto al límite inferior de referencia para la concentración de espermatozoides 15 millones por $\mathrm{mL}$, encontrando que se debe volver a establecer un valor mínimo de 20 millones por $\mathrm{mL}$. Skakkebaek (2010), es mucho más crítico y sugiere lo más pronto posible una nueva versión del manual OMS, para estandarización del semen humano, en el cual se aumente los valores de concentración espermática de 15 a 40 millones de espermatozoides por $\mathrm{mL}$, ya que la fecundidad masculina decrece progresivamente bajo estos valores. Además de considerar subir las formas normales (4\%), usando criterios estrictos, a límites referenciales más altos de $9 \%$ a $12 \%$.

Aún así todos los especialistas reconocen la importancia de realizar el análisis seminal como un primer indicador de fertilidad masculina, pero estiman conveniente el incorporar nuevos procedimientos en la evaluación de la capacidad funcional de los espermatozoides, tales como la integridad de ADN espermático, ya que el ADN alterado es muy frecuente en pacientes no fértiles (Góngora-Rodríguez \& Fontecilla-Ramírez; Cruz et al.; Horta et al.; García-Peiró et al., 2011).

En salud ambiental, debido al aumento de influencias negativas en el medio ambiente, el estudio de los parámetros seminales se considera un importante marcador biológico para evaluar la magnitud de las exposiciones a diferentes factores químicos ambientales y laborales, desta- cándose como un método de medición no invasivo (Espinoza-Navarro et al., 2010; Imai et al.; Ellekilde Bonde).

En conclusión la calidad del semen resulta ser la herramienta fundamental en la investigación masculina. Sin embargo los nuevos criterios de límites inferiores de referencia, aun resultan controversiales.

La mayoría de los autores proponen más y mejores instancias de controles de calidad (Interno y Externo), incorporando a personal calificado en la toma y procesamiento de las muestras.

Se recomienda incorporar otros procedimientos para evaluar efectivamente la capacidad funcional de los espermatozoides, tales como el estudio del ADN espermático.

El $5^{\circ}$ Manual para el examen y el procesamiento del semen humano (WHO, 2010), es posible que permita estandarizar mundialmente los resultados obtenidos en los diferentes centros, clínicas o laboratorios de reproducción, sin embargo muchos autores recomiendan una nueva revisión aumentando los parámetros de concentración, motilidad y morfología, de tal manera de poder determinar claramente población masculina fértil de población masculina subfertil.

\section{AGRADECIMIENTOS}

El trabajo fue financiado por la Universidad de Tarapacá (Arica-Chile) Proyecto de Investigación de la UTA: $\mathrm{N}^{\circ} 4710-11$.

ESPINOZA-NAVARRO, O. \& SARABIA, L. Evaluation and standardization of the quality of semen: new lower reference limit. Int. J. Morphol., 29(3):885-890, 2011.

SUMMARY: Spermogram or semen analysis is one of the most used parameters in the evaluation of male fertility. WHO (2010) presented the 5th Manual for review and processing of human semen, a document that was discussed during the first workshop of standardization of semen analysis (PLEAS), held in Santiago de Chile, May 2010. Subsequently applied the new values expressed in "lower reference limit" (LRL) for semen in several analysis studies conducted by various authors (2003 to 2010). The results indicate that $81 \%$ of Latin American researchers believe the new manual standardizes best sperm concentration, 96\% agree with the new subclassification in progressive sperm motility (A), non-progressive (B) and immobile (C). 68\% determined that the best instruments for the sperm count is the Neubauer haemocytometer chamber. Regarding quality control 
only $18 \%$ performed external quality control. $100 \%$ of researchers believe it is appropriate to conduct ongoing standardization workshops. Regarding the application of LRL in the study populations (2003-2010), 100\% comply with the standards to be considered a population with fertility capacity. However, several authors argue that a new version of the WHO manual, must be re-done urgently to better standardize sperm concentration (15 million/mL) and morphology according to strict criteria (4\%), reference values considered very low.

KEY WORDS: Semen analysis; Spermiogram; Male fertility; Lower reference limit.

\section{REFERENCIAS BIBLIOGRÁFICAS}

Aitken, R. J. Whither must spermatozoa wander? The future of laboratory seminology. Asian J. Androl., 12(1):99$103,2010$.

Agarwal, A. \& Said, T. Interpretation of basic semen analysis and advanced semen testing. In: Male Infertility: Problems and Solutions (Current Clinical Urology). Sabanegh, E. S. (Ed.). New York, Humana Press, 2011. pp.15-22.

Aulesa, C.; Lasheras, J.; Gris, J.; Herrero, J.; Márquez, C. \& Iglesias, A. Estudio de las variaciones estacionales de los parámetros analíticos del seminograma. Rev. Int. Androl., 5(4):337-42, 2007.

Cánovas, J.; Cadenas, V.; Gasset, R.; Fernández, J.; Sanchez, A. \& Garcia, J. Relación entre la edad y la calidad del estudio seminal. Experiencia en el área sanitaria 14 de la agencia valenciana de la salud. Arch. Esp. Urol., 61(6):705-10, 2008.

Cardona Maya, W. Manual de procesamiento de semen humano de la Organización Mundial de la Salud-2010. Actas Urol. Esp., 34(7):577-8, 2010.

Chen, Z.; Toth, T.; Godfrey-Bailey, L.; Mercedat, N.; Schiff, I. \& Hauser, R. Seasonal variation and age-related changes in human semen parameters. J. Androl., 24:22631, 2003.

Cooper, T. G.; Noonan, E.; von Eckardstein, S.; Auger, J.; Baker, H. W.; Behre, H. M.; Haugen, T. B.; Kruger, T.; Wang, C.; Mbizvo, M. T. \& Vogelsong, K. M. World Health Organization reference values of human semen characteristics. Hum. Reprod. Update, 16(3):231-45, 2010.
Cortés-Gutiérrez, E.; Dávila-Rodríguez, M.; LópezFernández, C.; Fernández, J. \& Gosálves, J. Evaluación del daño en el DNA espermático. Actas Urol. Esp., 31(2):120-31, 2007.

Crazzolara, S.; Wunder, D.; Nägeli, E.; Bodmer, C.; Graf, S. \& Birkhäuser, M.H. Semen parameters in a fertile Swiss population. Swiss Med. Wkly., 137(11-12):16672, 2007.

Cruz, I.; Colmenares, M.; Berrueta-Carrillo, L.; GomezPerez, R.; Montes, H.; Berrueta, L.; Salmen, S. \& Osuna, J. A. Evaluación de la calidad del espermatozoide humano: comparación entre la integridad del ADN espermático y variables del semen. Invest. Clin., 51(1):87-99, 2010.

Eliasson, R. Semen analysis with regard to sperm number, sperm morphology and functional aspects. Asian $J$. Androl., 12(1):26-32, 2010.

Ellekilde Bonde, J. P. Semen analysis from an epidemiologic perspective. Asian J. Androl., 12(1):91-4, 2010.

Espinoza-Navarro, O.; Cortés, S.; Monreal, J. \& Ferreccio, C. Análisis de las variables en jóvenes sanos en AricaChile. Rev. Méd. Chil., 138(12):1510-6, 2010.

Gao, J.; Gao, E. S.; Yang, Q.; Walker, M.; Wu, J. Q.; Zhou, W. J. \& Wen, S. W. Semen quality in a residential, geographic and age representative sample of healthy Chinese men. Hum. Reprod., 22(2):477-84, 2007.

García-Peiró, A.; Oliver-Bonet, M.; Navarro, J.; Abad, C.; Guitart, M.; Amengual, M. J. \& Benet, J. Sperm DNA integrity and meiotic behavior assessment in an infertile male carrier of a $9 \mathrm{qh}+++$ polymorphism. J. Biomed. Biotechnol., 2011:730847, 2011.

Góngora-Rodríguez, A. \& Fontecilla-Ramírez, D. La fragmentación de ADN espermático, influencia sobre las técnicas de reproducción asistida y la calidad embrionaria. Rev. Colomb. Obstet. Ginecolog., 16(2):160-4, 2010.

Ho, L. M.; Lim, A. S.; Lim, T. H.; Hum, S. C.; Yu, S. L. \& Kruger, T. F. Correlation between semen parameters and the Hamster Egg Penetration Test (HEPT) among fertile and subfertile men in Singapore. J. Androl., 28(1):15863, 2007.

Horta, F.; Madariaga, M.; García, A.; Hartel, S. \& Smith, R. Aumento del daño en el DNA espermático en varones mayores de 40 años. Rev. Med. Chile, 139(3), 2011. In press. 
Imai, A.; Ichigo, S.; Takagi, H.; Matsunami, K.; Suzuki, N. \& Yamamoto, A. Effects of cola intake on fertility: a review. Health, 2(9):997-1001, 2010.

Jensen, T. K.; Jørgensen, N.; Asklund, C.; Carlsen, E.; Holm, M. \& Skakkebaek, N. E. Fertility treatment and reproductive health of male offspring: A study of 1,925 young men from the general population. Am. J. Epidemiol., 165(5):583-90, 2007.

Levitas, E.; Lunenfeld, E.; Weisz, N.; Friger, M. \& Potashnik, G. Relationship between age and semen parameters in men with normal sperm concentration: analysis of 6022 semen samples. Andrologia, 39(2):45-50, 2007.

Lu, J. C.; Zhang, H. Y.; Hu, Y. A.; Huang, Y. F. \& Lü, N. Q. A survey on the status of semen analysis in 118 laboratories in China. Asian J. Androl., 12(1):104-10, 2010 .

MacLeod, J. \& Gold, R. Z. The male factor in fertility and infertility. VI. Semen quality and certain other factors in relation to ease of conception. Fertil. Steril., 4(1):1033, 1953.

Martini, A. C.; Molina, R. I.; Estofán, D.; Senestrari, D.; Fiol de Cuneo, M. \& Ruiz, R. D. Effects of alcohol and cigarette consumption on human seminal quality. Fertil. Steril., 82(2):374-7, 2004.

Menkveld, R. Clinical significance of low WHO sperm morphology value as proposed in the fifth edition of the WHO Laboratory Manual for the examination and processing of human semen. Asian J. Androl., 12(1):47$58,2010$.

Pacey, A. A. Quality assurance and quality control in the laboratory andrology. Asian J. Androl., 12(1):21-5, 2010.

Ramlau-Hansen, C. H.; Thulstrup, A. M.; Aggerholm, A. S.; Jensen, M. S.; Toft, G. \& Bonde, J. P. Is smoking a risk factor for decreased semen quality? A cross-sectional analysis. Hum. Reprod., 22(1):188-96, 2007.

Sarabia, L. \& Munuce, M. Nuevos valores para el espermiograma OMS 2010. Rev. Méd. Chile, 139(4), 2011. In press.

Skakkebaek, N. E. Normal reference range for semen quality and their relations to fecundity. Asian J. Androl., 12(1):95-8, 2010.

World Health Organization (WHO) Laboratory manual for the examination and processing of human semen and sperm-cervical mucus interaction. $4^{\text {th }}$ Ed. Cambridge, University Press, 1999.

World Health Organization (WHO) Laboratory manual for the examination and processing of human semen. $5^{\text {th }}$ Edition. Geneva, World Health Organization, 2010.

Dirección para correspondencia:

Prof. Dr. Omar Espinoza-Navarro.

Laboratory of Biology of Reproduction and Development. Universidad de Tarapacá.

Casilla 7/D

Velásquez 1775

Arica

CHILE

Teléfono: 5658205415.

Fax: 5658205484.

Email: oespinoz@uta.cl

Received: 20-05-2011

Accepted: 28-06-2011 